\title{
Emergency stenting for acute symptomatic carotid stenosis: dissecting the evidence
}

David J Werring ${ }^{1}$ and Fergus J Robertson ${ }^{2}$

${ }^{1}$ Stroke Research Group, Department of Brain Repair and Rehabilitation, UCL Institute of Neurology, Queen Square, London WC1N 3BG, United Kingdom

2Lysholm Department of Neuroradiology, National Hospital for Neurology and Neurosurgery, Queen Square, London WC1N 3BG, United Kingdom

Corresponding author: Dr David Werring

Stroke Research Group, Department of Brain Repair and Rehabilitation, UCL Institute of Neurology, Box 6, National Hospital for Neurology and Neurosurgery, Queen Square, London WC1N 3BG.

Tel: +44 (0)2034483541

Email: d.werring@ucl.ac.uk

Fax: +44 (0)2078338613

Keywords: stroke, carotid dissection, interventional neuroradiology

Word count: 697 excluding title page and references 
Most neurologists, vascular neuroradiologists and stroke physicians encounter carotid artery dissection (CAD) regularly, yet its management remains challenging. The main goal is prevention of ischaemic stroke in the territory of the affected artery, usually due to thromboembolism, but also potentially due to critically reduced flow in the dissected arterial segment, so-called haemodynamic stroke. Unfortunately, randomized controlled trial evidence is not available regarding the optimal medical treatment of extracranial dissection: antiplatelet agents or anticoagulants are both considered reasonable options (1).

Why is carotid dissection still a treatment challenge? First, like stroke itself, carotid dissection is not one disease. The aetiology (traumatic versus spontaneous) site of dissection (intracranial versus extracranial), degree of luminal stenosis and intracranial collateral circulation varies from patient to patient, with profound effects on risk, distribution and extent of resultant cerebral infarction. Second, although CAD accounts for about $20 \%$ of ischaemic strokes in younger people, recent randomized trials in CAD had major recruitment difficulties (1), suggesting that a definitive answer regarding optimum medical treatment is unlikely to emerge in the foreseeable future.

Where patients with CAD have threatened or recurrent secondary thromboembolic or haemodynamic cerebral ischaemia despite adequate medical therapy, or where medical treatment is contraindicated, there is considerable interest in endovascular treatments. Similarly to endovascular treatment of atherosclerotic carotid stenosis, a transarterial stent may be inserted with the aim of either restoring adequate luminal calibre or stabilizing a carotid pseudoaneurysm (itself a potential source of thromboemolism). These techniques are well established, with first reported carotid stent treatment for arterial dissection by Matsuura et al in 1997 (2). High technical success rates have been reported in a number of subsequent small case series, well reviewed by Xianjun et al in 2013 (3). There are specific technical challenges in selecting the true lumen in the dissected vessel and in preventing distal embolisation of thrombus from the dissection flap. Current data suggests a procedural morbidity of approximately $5 \%(3,4,5)$. Such studies seem liable to selection or publication bias, however, and true complication rates may be higher.

In this issue of Practical Neurology, Korya and colleagues describe a case of extracranial carotid dissection with recurrent transient symptoms in the affected hemisphere, which the authors convincingly suggest were due to haemodynamic failure (i.e. reduced perfusion) resulting from the tight carotid artery stenosis. The patient had experienced transient focal neurological symptoms, related to posture and with a low systemic blood pressure, supporting the idea of reduced cerebral perfusion, rather than thromboembolism, as a mechanism. The patient's symptoms stopped after a radiologically successful stenting procedure.

Although there is very limited high quality evidence for the effectiveness of acute carotid artery stenting in dissection, this case highlights its potential role in carefully selected cases where haemodynamic ischaemia seems to be the dominant problem. Of course, we can never know what would have happened 
without endovascular treatment, and given the rarity of this clinical situation, a randomized controlled trial will not be possible.

This case illustrates the challenges of treating uncommon cases in the field of stroke medicine. If a strictly evidence-based approach is taken, this patient should be managed with antithrombotic treatment only (either antiplatelets or anticoagulants). However, in this particular case, there was strong evidence for a haemodynamic mechanism for the recurrent symptoms, presumably with a substantial risk of early ischaemic stroke (although this is difficult to quantify) (6). The patient's attacks had continued despite antithrombotic therapy, and logical reasoning suggests that escalating the antithrombotic treatment further will not help the situation. In such exceptional cases, it seems reasonable to consider endovascular treatment.

But it is important that both doctors and patients appreciate the uncertainties and hazards of this course. Every effort should be made to systematically collect data on outcomes from such interventions, but the multidisciplinary approach to endovascular treatment can make this challenging; in the UK, for example, several carotid stent databases have been initiated in recent years, but none are currently comprehensive. Prospective outcome data should ideally be collected on all neuro-endovascular procedures undertaken outside randomized trials.

Although "do no harm" is a good starting point for all treatment decisions, this case report reminds us that in rarer clinical situations, evidence-based medicine will not always give the answer, and that careful consideration of the pathophysiology, and technical skill, can lead to a good outcome.

\section{References}

1 Kennedy F, Lanfranconi S, Hicks C, et al. Antiplatelets vs anticoagulation for dissection: CADISS nonrandomized arm and meta-analysis. Neurology 2012;79:686-689.

2. Matsuura JH, Rosenthal D, Jerius $\mathrm{H}$, et al: Traumatic carotid artery dissection and pseudoaneurysm treated with endovascular coils and stent. J Vasc Surg 1997; 4: 339-343.

3. Xianjun $\mathrm{H}$, Zhiming Z. A systematic review of endovascular management of internal carotid artery dissections. Interv Neurol. 2013 Sep;1(3-4):164-70.

4. Cohen JE, Gomori JM, Itshayek E, et al: Single-center experience on endovascular reconstruction of traumatic internal carotid artery dissection. J Trauma Acute Care Surg 2012; 72: 216-221.

5. Ohta H, Natarajan SK, Hauck EF, et al: Endovascular stent therapy for extracranial and intracranial carotid artery dissection: single-center experience. J Neurosurg 2011; 115: 91-100. 
6. Klijn CJ, Kappelle LJ. Haemodynamic stroke: clinical features, prognosis, and management. Lancet neurology 2010;9:1008-1017. 


\section{Contributorship}

Dr Werring wrote the first draft. Dr Robertson reviewed the literature and made critical revisions, and approved the final version.

\section{Funding}

This work was undertaken at UCLH/UCL who received a proportion of funding from the Department of Health's NIHR Biomedical Research Centres funding scheme.

\section{Competing interests}

None. 